\title{
Isolated osteonecrosis revealing primary antiphospholipid syndrome
}

\begin{abstract}
The orthopedic manifestations associated with primary antiphospholipid syndrome (APS) are very rare and little known. Among these manifestations, bone necrosis or avascular necrosis of bone remains exceptional and unusual: noted only in $0.9 \%$ of cases in the largest series of primary APS. Only a few sporadic cases of osteonecrosis as the first manifestations revealing this syndrome have been reported in the world literature. We report an original observation of isolated bone infarction of the upper end of the humerus revealing a primary APS in a 39-year-old woman.
\end{abstract}

Keywords: Osteonecrosis, Bone infarction, Primary antiphospholipid syndrome, Antiphospholipid antibodies, Avascular necrosis of bone
Volume II Issue I - 2019

\author{
Salem Bouomrani, ${ }^{1,2}$ Moez Ben Ayed ${ }^{2,3}$ \\ 'Department of Internal Medicine, Military Hospital of Gabes, \\ Tunisia \\ ${ }^{2}$ Sfax Faculty of Medicine, University of Sfax, Tunisia \\ ${ }^{3}$ Department of Orthopedics, Regional Hospital of Gabes, \\ Tunisia
}

Correspondence: Salem Bouomrani, Department of Interna Medicine, Military Hospital of Gabes, Gabes 6000, Tunisia, Tel +0021698977555, Email salembouomrani@yahoo.fr

Received: February II, 2019 | Published: February 14, 2019

\section{Introduction}

First described in 1983 by Graham RV Hughes, ${ }^{1}$ antiphospholipid syndrome (APS) is a clinical-biological entity characterized by the association of thromboembolic events, classically recurrent, with obstetrical complications, and the permanent and significant presence of autoantibodies against the phospholipids of cell membranes. ${ }^{2,3}$ It is a rare entity that may be primitive or secondary to other autoimmune diseases, in particular systemic lupus erythematosus. Its incidence is estimated at 5/100,000 person-years and its prevalence at 40 $50 / 100,000$ people. ${ }^{2,3}$ The clinical manifestations of this syndrome are highly polymorphic and all tissues and organs can be affected ${ }^{2-4}$ making its diagnosis a real challenge for clinicians. ${ }^{5}$ The orthopedic complications associated with primary APS are very rare and little known., 5 Among these manifestations, bone necrosis remains exceptional and unusual. $5,7,8$ We report an original observation of isolated osteonecrosis of the upper end of the humerus revealing a primary APS.

\section{Case report}

A 39-year-old woman, without pathological medical history, was consulting our day hospital for acute pain of the right shoulder evolving for a week and not improved by the analgesic treatment prescribed by his family doctor. The somatic examination noted minimal, anterior, and localized pain of the upper end of the right humerus. This pain was awakened by active mobilization of the right upper limb. The shoulder joint was supple, painless at mobilization, and with preserved mobility sector. There was no evidence of local inflammatory signs, ecchymosis, or right axillary lymphadenopathy. The rest of the somatic examination was without significant abnormalities.

The radiograph of the right shoulder showed the existence of an extensive irregular, central, sclerotic, and heterogeneous lesion of the upper end of the humerus with serpiginous contours (Figure 1). The cortical bone was respected as well as the glenohumeral joint. CTscan confirmed the same findings of the standard radiograph (Figure 2) compatible with the diagnosis of a bone infarct of the superior end of the right humerus (Figure 3). No other similar lesions were noted at other bone sites.

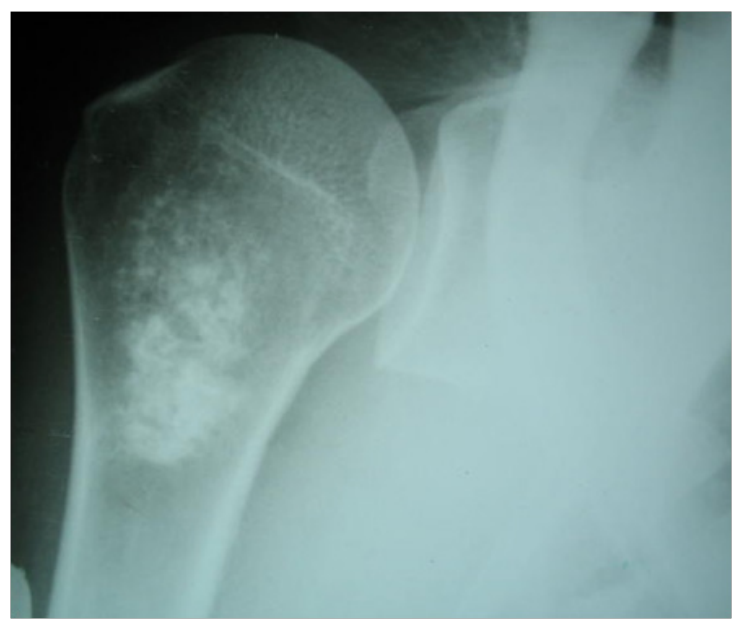

Figure I X-ray of the right shoulder: extensive irregular, sclerotic, and heterogeneous lesion of the upper end of the right humerus with serpiginous contours.

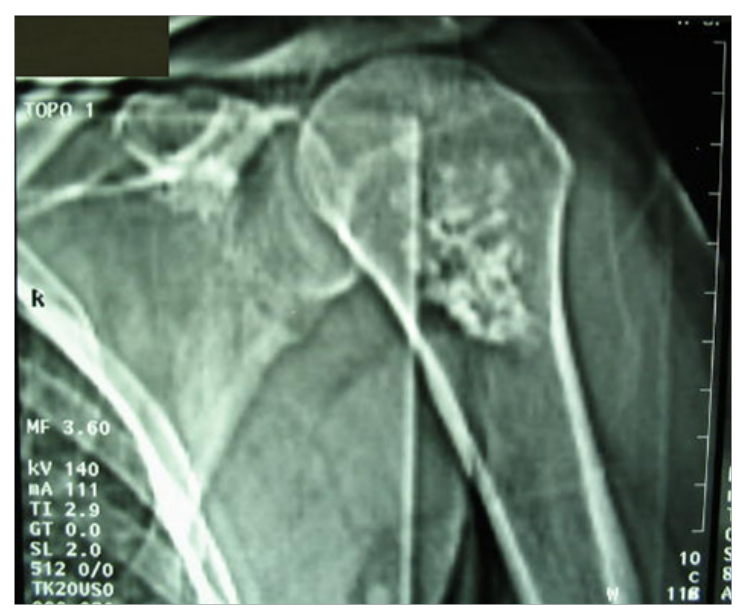

Figure 2 CT-scan of the right shoulder (Topogram): heterogeneous, central, and extensive opacity of the upper extremity of the right humerus with spicules. Bone corticals and articular surfaces are respected. 


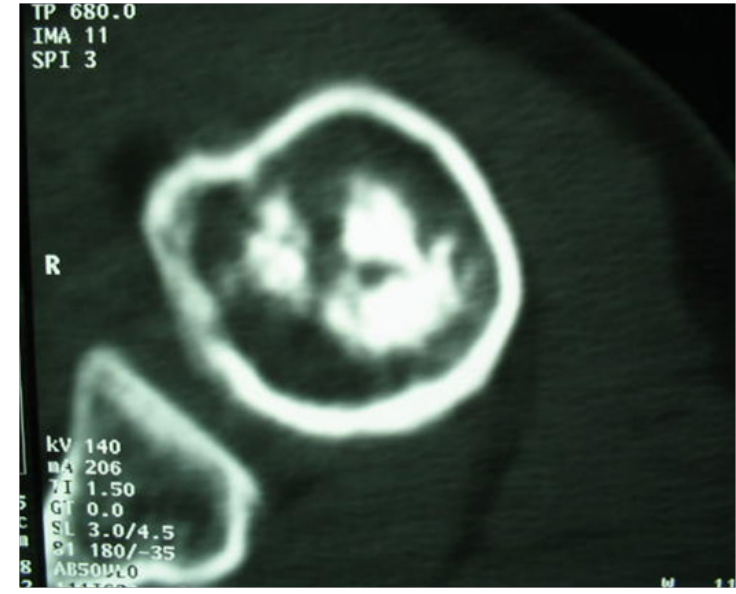

Figure $3 \mathrm{CT}$ of the right shoulder (axial section): bone infarction of the upper end of the right humerus.

The basic bioassays noted isolated thrombocytopenia at 115,000 / $\mathrm{mm}^{3}$; other tests have been in the normal range: hemoglobin, leukocytes, erythrocyte sedimentation rate, C-reactive protein, calcium, phosphoremia, alkaline phosphatase, creatinine, fasting glucose, uric acid, ionogram, transaminases, muscle enzymes, lactate dehydrogenase, thyroid tests, and electrophoresis of serum proteins.

The etiological assessment of this bone infarction was negative: in particular, there was no notion of trauma, chronic alcoholism or toxic substances consumption, and the patient had no cardiovascular/ atherosclerosis risk factors. Similarly, there was no underlying malignant hemopathy or cancer. The presence of thrombocytopenia controlled in several blood counts led to the realization of immunological investigations: the anti-nuclear and anti- native DNA antibodies were negative as well as the lupus anticoagulant. The antiphospholipid antibodies were strongly positive: anti-cardiolipin $\mathrm{IgG}$ at $56 \mathrm{AU} / \mathrm{ml}(\mathrm{Nl}<12)$ and anti- $\beta 2$-glucoprotein-1 ( $\beta 2 \mathrm{GP}-1)$ IgG at $42 \mathrm{GPL} / \mathrm{ml}(\mathrm{Nl}<10)$. Screening for other constitutional thrombophilias was negative (protein $\mathrm{C}, \mathrm{S}$, antithrombin III, and activated protein $\mathrm{C}$ resistance/Factor V-Leiden).

At the end of these investigations, the diagnosis of an isolated bone infarction of the upper end of the humerus revealing a primary APS was retained. The patient was treated with hydration, effective anticoagulation, and systemic corticosteroid therapy at a dose of 1 $\mathrm{mg} / \mathrm{kg}$ /day with a favorable evolution. Biological control after two months showed that anti-cardiolipin and anti- $\beta 2 \mathrm{GP}-1$ antibodies were still positive confirming the diagnosis of primary APS. No new thromboembolic event was noted for three years.

\section{Discussion}

The musculoskeletal manifestations reported during APS included: arthritis/arthritis, bone narrow necrosis, muscle infarction, complex regional pain syndrome type-1, non-traumatic fractures, avascular necrosis of bone, and osteoporosis., ${ }^{4,6}$ Orthopedic involvement associated with APS is still under-recognized and not well documented feature of this syndrome. ${ }^{6,710}$ It is therefore described as "novel" and "unusual" by the majority of authors. ${ }^{6,8,9,11}$ Osteonecrosis or avascular necrosis of bone, caused by thrombosis of terminal arteries in subchondral bone, remains an exceptional complication of primary APS. ${ }^{7,8,12,13}$ Indeed, in the largest series of 538 patients with a primary APS of Cervera R et al., ${ }^{14}$ osteonecrosis was noted only in $0.9 \%$ of cases. ${ }^{14}$ These bone infarctions are significantly more frequent during secondary APS associated with systemic lupus erythematosus; Systemic corticosteroid therapy, often prescribed at high doses during lupus, plays a role in the pathogenesis of these infarcts. ${ }^{15-17}$

Bone infarction in primary APS can be isolated, but most often is associated with other thromboembolic manifestations of the disease (cutaneous, cerebral, intestinal, hepatic, ocular, cardiac, renal, etc.). ${ }^{16,18}$ In the most severe forms of the disease, osteonecrosis fits into a clinical picture of multiorgan damage defining the catastrophic syndrome of antiphospholipids. ${ }^{18}$ Bone infarction associated with primary APS can be uni-, bilateral, or multiple (involving three or more bone anatomic sites). . $, 7,8,12,17,19$ Several sites of avascular osteonecrosis have been reported as sporadic cases during this syndrome: ${ }^{4}$ vertebrae, ${ }^{16}$ flat bones of the feet and wrists, ${ }^{15}$ femurs,,${ }^{7,8,15,17}$ tibia, ${ }^{8,17}$ humerus, ${ }^{7}$ and ribs. ${ }^{20}$ The revealing forms of the disease remain exceptional. ${ }^{7,12,19}$

Localized pain is the main symptom of osteonecrosis but this infarction can remain totally asymptomatic and would be incidentally discovered on imaging. ${ }^{4,7,21}$ MRI is the gold standard for the detection of bone infarctions associated with primary APS..$^{21}$ The treatment is in most cases conservative, based on hydration, effective anticoagulation, and systemic corticosteroids. Immunosuppressants are indicated in forms associated with other specific visceral involvement of the diseases. Surgical treatment is indicated in complicated forms. ${ }^{4,6,13,21}$

\section{Conclusion}

Bone infarction remains an exceptional and unusual complication during primary APS. The revealing forms represent a real diagnostic challenge for clinicians. Our observation is characterized by its isolated character, revealing presentation of the disease, and the absence of other contributing factors, in particular previous systemic corticosteroids. As rare as it is, this possible complication of primary APS deserves to be known by health professionals and the diagnosis of primary APS must be evoked in front of any bone infarction that is not proven, especially in young woman.

\section{Acknowledgements}

None.

\section{Conflicts of interest}

None.

\section{References}

1. Hughes GR. Thrombosis, abortion, cerebral disease, and the lupus anticoagulant. Br Med J (Clin Res Ed). 1983;287(6399):1088-1089.

2. Cervera R. Antiphospholipid syndrome. Thromb Res. 2017;151(Supp1 1):S43-S47.

3. Gómez-Puerta JA, Cervera R. Diagnosis and classification of the antiphospholipid syndrome. J Autoimmun. 2014;48-49:20-25.

4. Noureldine MH, Khamashta MA, Merashli M, et al.. Musculoskeletal manifestations of the antiphospholipid syndrome. Lupus. $2016 ; 25(5): 451-462$

5. Mallhi RS, Kushwaha N, Chatterjee T, et al. Antiphospholipid syndrome: A diagnostic challenge. Med J Armed Forces India. 2016;72(Suppl 1):S31-S36

6. Gorshtein A, Levy Y. Orthopedic involvement in antiphospholipid syndrome. Clin Rev Allergy Immunol. 2007;32(2):167-171.

7. Rueda JC, Duque MA, Mantilla RD, et al. Osteonecrosis and antiphospholipid syndrome. J Clin Rheumatol. 2009;15(3):130-132. 
8. Sciascia S, Bertero MT. Osteonecrosis and antiphospholipid antibodies Intern Med. 2009;48(9):737-738.

9. Hughes G. Hughes Syndrome: the antiphospholipid syndrome--a clinical overview. Clin Rev Allergy Immunol. 2007;32(1):3-12.

10. Hughes GR. Hughes Syndrome (the antiphospholipid syndrome): ten clinical lessons. Autoimmun Rev. 2008;7(3):262-266.

11. Asherson RA, Cervera R. Unusual manifestations of the antiphospholipid syndrome. Clin Rev Allergy Immunol. 2003;25(1):61-78.

12. Legrève S, Brands G1. Multifocal osteonecrosis revealing an antiphospholipid syndrome: case report. Rev Med Liege. 2017;72(5):232236.

13. Seleznick MJ, Silveira LH, Espinoza LR. Avascular necrosis associated with anticardiolipin antibodies. J Rheumatol. 1991;18(9):1416-1417.

14. Cervera R, Piette JC, Font J, et al. Antiphospholipid syndrome: clinical and immunologic manifestations and patterns of disease expression in a cohort of 1,000 patients. Arthritis Rheum. 2002;46(4):1019-1027.

15. Chatterjee $\mathrm{S}$. Bone infarcts in a woman with systemic lupus erythematosus and antiphospholipid antibody syndrome. CMAJ. 2006;174(4):455-456.
16. Sundaram PS, Padma S. Hughes syndrome with cerebral, skeletal infarction \& retinal vein thrombosis. Indian J Med Res. 2015;141(2):249250 .

17. Perez-Pampin E, Mera A, Campos J. Bone infarctions in a patient with systemic lupus erythematosus and anti-cardiolipin antibodies. J Clin Rheumatol. 2010;16(1):54.

18. Koenig M, Roy M, Baccot S, et al. Thrombotic microangiopathy with liver, gut, and bone infarction (catastrophic antiphospholipid syndrome) associated with HELLP syndrome. Clin Rheumatol. 2005;24(2):166168 .

19. Rostom S, Bahiri R, Hajjaj-Hassouni N. Multifocal avascular necrosis revealing antiphospholipid syndrome. Clin Rheumatol. 2008;27(2):271272.

20. Yoo WH. Multiple rib infarcts: a rare form of osteonecrosis in antiphospholipid syndrome. Ann Rheum Dis. 2004;63(4):457-458.

21. TektonidouMG, MalagariK, Vlachoyiannopoulos PG, etal.Asymptomatic avascular necrosis in patients with primary antiphospholipid syndrome in the absence of corticosteroid use: a prospective study by magnetic resonance imaging. Arthritis Rheum. 2003;48(3):732-736. 